Homology, Homotopy and Applications, vol.4(2), 2002, pp.219-225

\title{
GROWTH AND LIE BRACKETS IN THE HOMOTOPY LIE
} ALGEBRA

\author{
YVES FÉLIX, STEPHEN HALPERIN AND JEAN-CLAUDE THOMAS
}

(communicated by Clas Löfwall)

Abstract

Let $L$ be an infinite dimensional graded Lie algebra that is either the homotopy Lie algebra $\pi_{*}(\Omega X) \otimes \mathbb{Q}$ for a finite $n$ dimensional CW complex $X$, or else the homotopy Lie algebra for a local noetherian commutative $\operatorname{ring} R\left(U L=\operatorname{Ext}_{R}(I k, I k)\right)$ in which case put $n=(\operatorname{embdim}-\operatorname{depth})(R)$.

Theorem: (i) The integers $\lambda_{k}=\sum_{q=k}^{k+n-2} \operatorname{dim} L_{i}$ grow faster than any polynomial in $k$.

(ii) For some finite sequence $x_{1}, \ldots, x_{d}$ of elements in $L$ and some $N$, any $y \in L_{\geqslant N}$ satisfies: some $\left[x_{i}, y\right] \neq 0$.

\section{To Jan-Erik Roos on his sixty-fifth birthday}

\section{Introduction}

Let $X$ be a simply connected CW complex of finite type. Then [16] its loop space homology, $H_{*}(\Omega X ; \mathbb{Q})$ is the universal enveloping algebra of the graded Lie algebra $L_{X}=\left\{\left(L_{X}\right)_{i}\right\}_{i \geqslant 1}=\pi_{*}(\Omega X) \otimes \mathbb{Q}$, equipped with the Samelson product. Similarly, if $R$ is a commutative local noetherian ring with residue field $I k$ then [1], [17] $\operatorname{Ext}_{R}(I k, I k)$ is the universal enveloping algebra of a graded Lie algebra $L_{R}=\left\{L_{R}^{i}\right\}_{i \geqslant 1}$. We call $L_{X}\left(L_{R}\right)$ the homotopy Lie algebra of $X($ of $R)$ and call $e_{i}(X)=\operatorname{dim}\left(L_{X}\right)_{i}\left(\right.$ or $\left.e_{i}(R)=\operatorname{dim} L_{R}^{i}\right)$ the $i^{\text {th }}$ deviation of $X$ (or of $R$ ).

For finite complexes $X$ and for all local $\operatorname{rings} R$ the hypothesis $\operatorname{dim} L<\infty$ imposes very special conditions (in this case $X$ is called $\mathbb{Q}$-elliptic). For example, if $X$ is $\mathbb{Q}$-elliptic the $H_{*}(\Omega X ; \mathbb{Q})$ is a Poincaré duality algebra [12] while if $L_{R}$ is finite dimensional then $R$ is a complete intersection [10], [11]. Moreover, it is known (again for finite complexes $X$ and for any $R$ ) that

- If $\operatorname{dim} L_{X}<\infty$ and $\operatorname{dim} L_{R}<\infty$ then

$$
e_{i}(X)=0, i \geqslant 2 \operatorname{dim} X,[\mathbf{9}] \quad \text { and } \quad e_{i}(R)=0, i \geqslant 3,[\mathbf{1 0}],[\mathbf{1 1}] .
$$

- If $\operatorname{dim} L_{X}=\infty$ and $\operatorname{dim} L_{R}=\infty$ then for some $K>0, C>1$,

Received January 8, 2001, revised September 17, 2001; published on July 12, 2002. 2000 Mathematics Subject Classification: 55P62, 55P35, 17B70, 16 L99.

Key words and phrases: Finite CW complex - Local ring - Homotopy Lie algebra - Depth.

(C) 2002, Yves Félix, Stephen Halperin and Jean-Claude Thomas. Permission to copy for private use granted. 


$$
\sum_{i=1}^{k} e_{i}(X) \geqslant K C^{k}, k \geqslant \operatorname{dim} X-1,[\mathbf{3}] \quad \text { and } \quad \sum_{i=1}^{k} e_{i}(R) \geqslant K C^{k}, k \geqslant 1,[\mathbf{2}] .
$$

- If $\operatorname{dim} L_{X}=\infty$ and $\operatorname{dim} L_{R}=\infty$ then

$$
\sum_{i=k}^{k+\operatorname{dim} X-2} e_{i}(X)>0 \text {, all } k \geqslant 1,[\mathbf{1 4}] \quad \text { and } \quad e_{k}(R)>0 \text {, all } k \geqslant 1,[\mathbf{1 3}] .
$$

- If $\operatorname{dim} L=\infty\left(L=L_{X}\right.$ or $\left.L_{R}\right)$ then for all $x \in L$ of sufficiently large even degree there is some $y=y(x) \in L$ such that $(a d x)^{k} y \neq 0, k \geqslant 1$, [7].

These results motivate/provide evidence for the two following main conjectures, due to some combination of Avramov - Félix - Halperin - Thomas.

Conjecture 1. If $X$ is finite dimensional, not $\mathbb{Q}$-elliptic, and if $R$ is not a complete intersection then for some $K>0, C>1$ :

$$
\sum_{i=k}^{k+\operatorname{dim} X-2} e_{i}(X) \geqslant K C^{k}, \quad k \geqslant 1, \quad \text { and } \quad e_{k}(R) \geqslant K C^{k}, \quad k \geqslant 1 .
$$

Conjecture 2. If $X$ is finite dimensional, not $\mathbb{Q}$-elliptic, and if $R$ is not a complete intersection then $L_{X}$ and $L_{R}$ each contain a free Lie subalgebra on two generators.

This paper makes some progress towards these conjectures. For simplicity we adopt the following notation:

- $X$ is a finite, non $\mathbb{Q}$-elliptic, simply connected $\mathrm{CW}$ complex and $R$ is a local noetherian commutative ring that is not a complete intersection.

- $L$ is either $L_{X}$ or $L_{R}$, and $L_{\text {even }}$ is the sub Lie algebra of elements of even degree.

- $n=n_{X}=\operatorname{dim} X$ or $n=n_{R}=($ emb dim- $\operatorname{depth})(R)$.

- $e_{i}=e_{i}(X)$, or $e_{i}=e_{i}(R)$.

- $h=h_{X}=\operatorname{dim} H^{*}(X ; \mathbb{Q})$, or $h=h_{R}=\operatorname{dim} H_{*}\left(K^{R}\right), K^{R}$ denoting the Koszul complex of $R$.

Then, with the hypotheses and notation above, we establish

\section{Theorem A.} ular,

(i) The integers $\lambda_{k}=\sum_{i=k}^{k+n-2} e_{k}$ grow faster than any polynomial in $k$. In partic-

$$
\lambda_{k} \rightarrow \infty \text { as } k \rightarrow \infty .
$$

(ii) Moreover, if $L_{\text {even }}$ contains a maximal abelian sub Lie algebra of finite dimension then for some $K>0, C>1$,

$$
\lambda_{k} \geqslant K C^{k}, \quad k \geqslant 1 \text {. }
$$

Theorem B. There is a finite sequence $x_{1}, \ldots, x_{d}$ of elements in $L$ and an integer $N$ such that:

$$
y \in L, \quad \operatorname{deg} y \geqslant N \Rightarrow \text { some }\left[x_{i}, y\right] \neq 0 .
$$




\section{General remarks.}

With $X$ is associated the commutative graded differential algebra $A_{P L}(X)$ whose Sullivan minimal model $(\Lambda V, d)$ satisfies $[\mathbf{1 8}],[\mathbf{8}]$

$$
H(\Lambda V, d) \cong H^{*}(X ; \mathbb{Q}) \quad \text { and } \quad e_{i}(X)=\operatorname{dim} V^{i+1}, i \geqslant 1 .
$$

In particular, $H^{i}(\Lambda V, d)=0, i>n$. Moreover, [18], [8] the differential $d=\sum_{i \geqslant 2} d_{i}$, with $d_{i}: V \rightarrow \Lambda^{i} V$. Finally, $\left(\Lambda V, d_{2}\right)=C^{*}\left(L_{X}\right)$ where for any graded Lie algebra $E$ over $I k, C^{*}(E)$ is the Cartan-Chevalley-Eilenberg-Quillen complex, whose cohomology is $\operatorname{Ext}_{U E}(I k, I k)$.

Similarly, with $R$ is associated its Koszul complex $K^{R}$ which is connected by quasi-isomorphisms to a commutative graded chain algebra [2]. This in turn has a 'Sullivan model' $(\Lambda V, d)$ in which $V=\left\{V_{i}\right\}_{i} \geqslant 1$ and $d$ decreases degrees by 1 . Here we have $H_{i}(\Lambda V, d)=H_{i}\left(K^{R}\right)=0, i>n$, and

$$
e_{i}(R)=\operatorname{dim} V_{i-1}, \quad i \geqslant 2 .
$$

Moreover $\left(\Lambda V, d_{2}\right)=C^{*}\left(L_{R}^{\geqslant 2}\right)$.

Recall now that the depth of an augmented graded algebra $A$ is the least $m$ (or $\infty)$ such that $\operatorname{Ext}_{A}^{m}(I k, A) \neq 0$. We define the depth of a graded Lie algebra, $E$, to be the depth of its universal enveloping algebra (depth $E=\operatorname{depth} U E$ ) and recall from $[4]$ that

$$
\operatorname{depth} L_{X} \leqslant \operatorname{LScat}(X) \leqslant n_{X} \quad \text { and depth } L_{R} \leqslant n_{R} \text {. }
$$

We shall make frequent use of the remark [4] that if $I$ is an ideal in a graded Lie algebra $E$ then

$$
\operatorname{depth} I \leqslant \operatorname{depth} E \text {. }
$$

Finally, since in both cases we have $\operatorname{dim} H(\Lambda V, d)=h<\infty$, we can apply a result of Lambrechts:

Lemma 2.3 [15]. For all $k$ sufficiently large, there is some $l \in[k+1, k+n-1]$ such that

$\operatorname{dim} V^{l} \geqslant \operatorname{dim} V^{k} / h n$.

In fact Lambrechts shows that $\operatorname{dim} V^{k} \leqslant h \sum_{i=1}^{n-1} \operatorname{dim} V^{k+i}+\operatorname{dim} G_{k}$, where $G_{*} \subset L$ is the abelian ideal of Gottlieb elements. As noted in [4] this implies that $G_{*}$ is finite dimensional, and so the inequality of Lemma 2.3 holds for large $k$.

\section{Proof of Theorem A.}

(i) We prove this in the case that $L_{\text {even }}$ contains an infinite dimensional abelian sub Lie algebra, $E$, since otherwise (i) will follow from (ii). For convenience, we abuse notation and write the degrees as subscripts. 
Note that the sub Lie algebra $F=E_{\leqslant k} \oplus L_{>k}$ has finite codimension in $L$. Thus we can write $F=I^{m} \subset I^{m-1} \subset \cdots \subset I^{0}=L$ where each $I^{k}$ is constructed from $I^{k+1}$ by adding a single element of maximal degree. It follows that each $I^{k}$ is a sub Lie algebra containing $I^{k+1}$ as an ideal. In particular by (2.1) and (2.2).

$$
\operatorname{depth} F \leqslant \operatorname{depth} L \leqslant n .
$$

On the other hand $F / L_{>k}$ is the abelian Lie algebra $E_{\leqslant k}$ and $U E_{\leqslant k}=I k\left[E_{\leqslant k}\right]$ is a polynomial algebra. In particular, depth $E_{\leqslant k}=\operatorname{dim} E_{\leqslant k}$, and there are constants $0<c<C$ such that for any finitely generated $U E_{\leqslant k}$-module $M$, and some integer $r(M)$,

$$
c k^{r(M)} \leqslant \sum_{i \leqslant k} \operatorname{dim} M_{i} \leqslant C k^{r(M)}, \quad k \text { sufficiently large. }
$$

The integer $r(M)$ is called the polynomial growth of $M$.

Now ([6];Theorem 4.1) asserts that for some $q \leqslant n$ and some $\alpha \in \operatorname{Tor}_{q}^{U L_{>k}}(I k, I k)$ the module $U E_{\leqslant k} \cdot \alpha$ has polynomial growth at least equal to $\left(\operatorname{dim} E_{\leqslant k}\right)-n$. But the action of $U E_{\leqslant k}$ in $\operatorname{Tor}^{U L_{>k}}(I k, I k)$ is induced from the adjoint representation of $E_{\leqslant k}$ in the complex $\left(\Lambda^{C}\left(s L_{>k}\right), \partial\right)$ dual to $C^{*}\left(L_{>k}\right)$; here $\Lambda^{C}$ denotes the free co-commutative coalgebra. In particular for some $z \in\left(\Lambda^{C}\right)^{q} s L_{>k}, U E_{\leqslant k} \cdot z$ has polynomial growth at least equal to $\left(\operatorname{dim} E_{\leqslant k}\right)-n$.

Since $q \leqslant n$ this implies in turn that for some $y \in L_{>k}$,

$$
\text { poly growth }\left(U E_{\leqslant k} \cdot y\right) \geqslant \frac{\operatorname{dim} E_{\leqslant k}}{n}-1 \text {. }
$$

Fix some $r>0$ and choose $k$ so that $\operatorname{dim} E_{\leqslant k} \geqslant(n+1) r$. Then poly growth $\left(U E_{\leqslant k} \cdot y\right) \geqslant r$. It follows that there are $r$ elements $x_{1}, \ldots, x_{r} \in E_{\leqslant k}$ such that $I k\left[x_{1}, \ldots, x_{r}\right] \stackrel{\cong}{\rightrightarrows} I k\left[x_{1}, \ldots, x_{r}\right] \cdot y$. Choosing $d_{i}$ so that the $x_{i}^{d_{i}}$ all have the same degree $d$ we see that

$$
\operatorname{dim} L_{k d+\operatorname{deg} y} \geqslant \lambda k^{r} \geqslant \mu((k+1) d+\operatorname{deg} y)^{r}, k \geqslant 2,
$$

for some positive constants $\lambda$ and $\mu$. Now, for $k$ sufficiently large, repeated applications of Lemma 2.3 give an infinite sequence of integers $i_{1}<i_{2}<\ldots$ such that $i_{1}=k d+\operatorname{deg} y$, and

$$
i_{s+1} \leqslant i_{s}+n-1 \text { and } \operatorname{dim} L_{i_{s}} \geqslant \frac{\mu((k+1) d+\operatorname{deg} y)^{r}}{(n h)^{s}}, \quad s \geqslant 1 .
$$

It follows at once that (provided $k$ is sufficiently large)

$$
\sum_{j=q}^{q+n-2} \operatorname{dim} L_{j} \geqslant \frac{\mu}{(n h)^{d}} q^{r}, \quad \operatorname{deg} y+k d \leqslant q \leqslant \operatorname{deg} y+(k+1) d .
$$

Since both sides of the equation are independent of $k$ this establishes (i) in the presence of an infinite dimensional abelian subalgebra.

(ii) Let $E=\oplus_{i=1}^{r} I k x_{i}$ be a maximal abelian sub Lie algebra of $L_{\text {even }}$. Give $L_{\text {even }}$ the decreasing filtration defined by $F^{0}=L_{\text {even }}$, and $F^{i}=\left\{y \in L_{\text {even }} \mid\left[x_{j}, y\right]=0, \quad 1 \leqslant\right.$ $j \leqslant i\}$. The maximality of $E$ implies that $F^{r}=0$. Choose graded subspaces $V^{i} \subset$ 
$L_{\text {even }}$ such that $F^{i-1}=V^{i} \oplus F^{i}$, and choose integers $d_{1}, \ldots, d_{r}$ so that $d_{1} \operatorname{deg} x_{1}=$ $\ldots=d_{r} \operatorname{deg} x_{r}=d$. Then for all $q$ and all $k$

$$
\left(a d x_{1}\right)^{q d_{1}} \oplus \ldots \oplus\left(a d x_{r}\right)^{q d_{r}}: V_{2 k}^{1} \oplus \ldots \oplus V_{2 k}^{r} \longrightarrow L_{2 k+q d}
$$

is injective ; i.e. $\operatorname{dim} L_{2 k} \leqslant \operatorname{dim} L_{2 k+q d}, k \geqslant 0, q \geqslant 0$.

On the other hand, a simple extension of the argument in ([8]; Chapter 33) gives an infinite sequence of even integers $i_{1}<i_{2}<\ldots$ such that $i_{s+1} \leqslant n^{2} i_{s}, s \geqslant 1$, and constants $a>0, D>1$ such that $\operatorname{dim} L_{i_{s}} \geqslant a D^{i_{s}}, s \geqslant 1$. Now application of Lambrecht's lemma 2.3 gives (ii) in the same way it completed the proof of (i).

\section{Proof of Theorem B.}

As recalled in $\S 2, L$ has finite depth. This means that $\operatorname{Ext}_{U L}(I k, U L) \neq 0$, and in [5] it is shown that for some finitely generated sub Hopf algebra $G$ the restriction $\operatorname{Ext}_{U L}(I k, U L) \rightarrow \operatorname{Ext}_{G}(I k, U L)$ is non zero. Suppose $G$ is generated in degrees less than or equal to $n$, and denote $E=L_{\leqslant n}$. Then the restriction $\operatorname{Ext}_{U L}(I k, U L) \rightarrow \operatorname{Ext}_{G}(I k, U L)$ factors through $\operatorname{Ext}_{U E}(I k, U L)$, and so the restriction $\operatorname{Ext}_{U L}(I k, U L) \rightarrow \operatorname{Ext}_{U E}(I k, U L)$ is non zero. In particular, $E$ has finite depth. The adjoint action of $E$ in $L$ defines a representation of $U E$ in $L$, and Theorem B is a corollary of

Theorem C. For some $N$ and all $y \in L \geqslant N$ the graded vector space $U E \cdot y$ grows faster than any polynomial.

Proof. Let $Z \subset L$ be the subspace of elements $z$ such that $U E \cdot z$ grows at most polynomially (i.e. for some constant $c>0$ and some $r$, $\operatorname{dim}[U E \cdot z]_{k} \leqslant c k^{r}, k \geqslant 1$. Since $U E \cdot[z, w] \subset[U E \cdot z, U E \cdot w]$ it follows that $Z$ is a sub Lie algebra of $L$, stable under the adjoint representation of $E$.

In particular, if $x \in Z \cap E$ then $U E . x$ is an ideal in $E$ of at most polynomial growth. Since depth $U E . x \leqslant$ depth $E<\infty$ (by 2.2) if follows from ([6]; Theorem B) that $U E . x$ is finite dimensional. Thus $Z \cap E$ is an ideal in $E$ that is the union of finite dimensional ideals. Since $L=L \geqslant 1$ these finite dimensional ideals are solvable and their sum $Z \cap E$ is then itself finite dimensional [4].

Thus $Z_{\geqslant q} \cap E=0$ (some $q$ ) and $E \oplus Z_{\geqslant q}$ is itself a sub Lie algebra of $L$. Moreover the composite

$$
\operatorname{Ext}_{U L}(I k, U L) \rightarrow \operatorname{Ext}_{U\left(E \oplus Z_{\geqslant q}\right)}(I k, U L) \rightarrow \operatorname{Ext}_{U E}(I k, U L)
$$

is non-zero. But $\operatorname{Ext}_{U\left(E \oplus Z Z_{q}\right)}(I k, U L)$ is the cohomology of the complex $\left(\wedge(s E)^{*} \otimes\right.$ $\left.\wedge\left(s Z_{\geqslant q}\right)^{*} \otimes U L, d\right)$, and a simple 'filtration argument' shows that the restriction to $\left(\wedge(s E)^{*} \otimes U L, d\right)$ is zero in cohomology unless for some $a \in U L, 1 \otimes a$ is a cocycle in the quotient complex $\left(\wedge\left(s Z_{\geqslant q}\right)^{*} \otimes U L, d\right)$. This can only occur when $Z_{\geqslant q}$ is finite dimensional and concentrated in odd degrees $[\mathbf{4}]$.

Thus $Z$ itself is finite dimensional and it suffices to choose $N$ so that $Z$ is concentrated in degrees $<N$. 


\section{References}

[1] M. André, Hopf algebras with divided powers, J. of Algebra, 18 (1976), 19-50.

[2] L.L. Avramov, Local algebra and rational homotopy, Astérisque 113-114, Société Mathématique de France (1984), 15-43.

[3] Y. Félix and S. Halperin, Rational $L S$ category and its applications, Trans. Amer. Math. Soc., 273 (1982), 1-37.

[4] Y. Félix and S. Halperin, C. Jacobson, C. Löfwall, J.-C. Thomas, The radical of the homotopy Lie algebra, Amer. J. of Math. 110 (1988), 301-322.

[5] Y. Félix, S. Halperin, and J.-C. Thomas, Hopf algebras of polynomial growth, Journal of Algebra, 125 (1989), 408-417.

[6] Y. Félix, S. Halperin, and J.-C. Thomas, Lie algebras of polynomial growth, J. London Math. Soc. 43 (1991), 556-566.

[7] Y. Félix, S. Halperin and J.-C. Thomas, Engel elements in the homotopy Lie algebra, J. of Algebra 144 (1991), 67-78.

[8] Y. Félix, S. Halperin and J.-C. Thomas, Rational Homotopy Theory, Graduate Text in Math. 205 Springer-Verlag 2000

[9] J. Friedlander and S. Halperin, An arithmetic characterization of the rational homotopy groups of certain spaces, Inventiones Math. 53 (1979), 117-133.

[10] T. H. Gulliksen, A homological charaterisation of local complete intersections, Compositio Mathematica 23 (1971), 251-255.

[11] T. H. Gulliksen, On the deviations of a local ring, Math. Scand. 47 (1980), 5-20.

[12] S. Halperin, Finiteness in the minimal models of Sullivan, Trans. Amer. Math. Soc. 230 (1977), 173-199.

[13] S. Halperin, The non-vanishing of the deviations of a local ring, Comment. Math. helvetici 62 (1987), 646-653.

[14] S. Halperin, Torsion gaps in the homotopy of finite complexes, II Topology 30 (1991), 471-478.

[15] P. Lambrechts, Analytic properties of Poincaré series of spaces, Topology 37 (1998), 1363-1370.

[16] J. Milnor and J.C. Moore, The structure of algebras, Annals of Math., 81 (1965), 211-264.

[17] G. Sjödin, Hopf algebras and derivations, J. Algebra 64 (1980), 218-229. 
[18] D. Sullivan, Infinitesimal computations in topology, Publ. Math. I.H.E.S. 47 (1977), 269-331.

This article may be accessed via WWW at http://www.rmi.acnet.ge/hha/ or by anonymous ftp at

ftp://ftp.rmi.acnet.ge/pub/hha/volumes/2002/n2a9/v4n2a9.(dvi,ps,pdf)

Yves Félix

Institut de Mathématiques,

Université de Louvain-La-Neuve,

B-1348 Louvain-La-Neuve,

Belgium.

Stephen Halperin

College of Computer, Mathematical and Physical Sciences, University of Maryland,

College Park, MD 20742-3281,

USA.

Jean-Claude Thomas thomas@tonton.univ-angers.fr

Facultd́es Sciences

Université d'Angers,

49045 bd Lavoisier, Angers,

France. 